\title{
Negotiating the Borders of the Gender Regime. Developments and Debates on Trans(sexuality) in the Federal Republic of Germany
}

Adrian de Silva

Bielefeld 2018: transcript Verlag, 436 Seiten.

Gundula Ludwig

Universität Bremen, Germany

E-Mail: gundula.ludwig@uni-bremen.de
Kürzlich vollzog sich im deutschen Rechtssystem mit Blick auf die rechtliche Absicherung von Zweigeschlechtlichkeit ein wichtiger Wandel: Seit I. Januar 2019 ist es intersexuellen Menschen möglich, den Geschlechtsantrag "divers" in das Personenstandsregister eintragen zu lassen. Unumkämpft ist diese Transformation jedoch freilich nicht: So bleibt weiterhin die Definitionsmacht über die Frage, was Geschlecht ist, im Kompetenzbereich der Medizin, denn Voraussetzung für den Eintrag ist die medizinische Diagnose einer ,Varianz der Geschlechtsentwicklung؛ Darüber hinaus beschränkt das Gesetz die vollzogene Öffnung insofern, als es den Personenkreis, dem die rechtliche Anerkennung eines diversen Geschlechts zugesprochen wird, auf intersexuelle Menschen begrenzt. Alle Menschen, die Geschlecht als divers definieren und leben und nicht als ,intersexuell' gelten, sehen sich weiterhin einem wirkmächtigen medizinisch-juridischen Macht-Wissens-Komplex gegenüber, der vorgibt, es gebe lediglich zwei Geschlechter.

Die erste umfassende Studie, wie seit den I960er Jahren bis 2014 ,Trans(Sexualität) ' im Recht und der Medizin in Deutschland verhandelt wurde, hat Adrian de Silva mit seiner Dissertation "Negotiating the Borders of the Gender Regime" vorgelegt. Obgleich die Studie interdisziplinär ausgerichtet ist, reiht sie sich auch in den noch jungen Zweig queer-theoretischer politikwissenschaftlicher Analysen ein, die zeigen, wie die Binarität von Körpern und Subjekten durch staatliche Institutionen reguliert, produziert und legitimiert wird. Gegenstand der Studie ist das Zusammenwirken von sexualwissenschaftlichen Diskursen über, Transsexualität' und ,Geschlechtsidentitätsstörungen', deren rechtliche Insti- tutionalisierung und ihre Umkämpftheit durch GegenWissen über Geschlecht und (Trans-)Körper von heterogenen Akteur*innen trans-aktivistischer Bewegungen.

Den Interaktionen zwischen den drei Feldern sowie ihrem historischen Wandel wird entlang von drei Fragekomplexen nachgegangen: „How did sexology, the law, the political branch of the trans movement and federal politics, mirrored in the practices and mediated by the procedures of the respective discipline and area, construct trans(sexuality) in relation to socially accepted genders?" (I7) Ausgangsannahme hierfür ist, dass naturalisierende Bestimmungen, was Geschlecht überhaupt ist, die Definition von Trans(Sexualität) maßgeblich formen. Auf der Prämisse aufbauend, dass alle drei Felder Arenen von Konflikten und Machtverhältnissen sind, wird des Weiteren untersucht, wie manche Vorstellungen von Gender hegemonial und andere marginalisiert wurden (I8). Schließlich beleuchtet de Silva, ,how and what concepts of trans(sexuality) entered other disciplines and fields and how concepts of trans(sexuality) were read into, or challenged in the respective parameters of the disciplines or social arenas in an uneven and frequently conflictual process" (ibid.). Diesen Fragen geht de Silva durch Analysen von sexualwissenschaftlichen Monographien und Zeitschriften, Gerichtsurteilen, rechtswissenschaftlichen Kommentaren, Kommentierungen und Stellungnahmen, Plenardebatten im Bundestag und Bundesrat sowie Stellungnahmen und Positionspapieren von transaktivistischen Gruppen nach.

Unterteilt ist die Studie in drei historische Blöcke: in den Zeitraum vor und während des Beschlusses des „Gesetz über die Änderung der Vornamen und die Feststellung der Geschlechtszugehörigkeit in besonderen 
Fällen“ - zumeist als ,Transsexuellen Gesetz' (TSG) abgekürzt; den Zeitrahmen von 1990 bis 20IO, in der das Gesetz sowie sexualwissenschaftliche Definitionen von Trans(Sexualität) mehrfache Veränderungen durchliefen; und in den Zeitraum von 20Io bis 20I4, für den Entwicklungen im Recht, den Sexualwissenschaften und TransPolitiken nach Einführung des TranssexuellenÄnderungsgesetz dargelegt werden.

Theoretisch eingebettet ist die Studie neben Trans und Queer Studies, die de Silvas epistemologische Grundlage von Geschlecht als machtvolle Konstruktion bilden, auch in (queer-)feministische staats- und hegemonietheoretische Ansätze, mit denen de Silva (Rechts-) Politiken zu Trans(Sexualität) als Materialisierung gesellschaftlicher Kräfteverhältnisse und Auseinandersetzungen heterogener Akteur*innen begreift.

Auf der Basis überaus detailreicher Rekonstruktionen wird dargelegt, dass sich im Laufe des Untersuchungszeitraums Vorstellungen von Geschlecht und Trans(Sexualität) partiell geöffnet haben: So wichen die naturalisierenden sowie heterosexuellen Prämissen von medizinischen wie rechtlichen ,Wahrheitsregimen von Geschlecht, (Geschlechts)Körpern und Identitäten auf, was in der Aufhebung von Zwangssterilisierung und Ehe-Scheidung im TSG deutlich wird. Diese Änderungen wurden freilich auch durch den wachsenden Einfluss von transaktivistischen Bewegungen erreicht. Zugleich führt de Silvas Studie jedoch die Beharrungstendenzen von Heteronormativität in Recht und Medizin vor, die sich nicht zuletzt schlicht daran zeigen, dass die rechtliche und medizinische Anerkennung der Fluidität und Nicht-Binarität von,Geschlecht' weiterhin als gesellschaftliches Randphänomen und Abweichung konstruiert wird und weiterhin „exclusively applied to individuals defined as transsexual" (382f.) bleibt.

Eine interdisziplinäre Studie über einen derart langen Zeitraum vorzulegen und dabei nicht nur die Felder Sexualwissenschaften, Recht und Soziale Bewegungen einzeln zu rekonstruieren, sondern in ihren Interaktionen darzustellen, macht den innovativen Charakter des Buches aus. De Silva ist es nicht nur gelungen, einen komplexen Gegenstand mit vielen Diskurssträngen zu bündeln, sondern diesen stets klar strukturiert darzustellen. Auf diese Weise stellt das Buch eine anregende, materialreiche Lektüre für Interessierte der (Trans) Gender Studies, Queer Theorie, Sexualwissenschaften, Soziologie sowie Rechts- und Politikwissenschaften dar. 